\title{
Ehrenpromotion Vietoris
}

Am 12. Juni 1994 verlieh die Universität Innsbruck das Ehrendoktorat der technischen Wissenschaften an das DMV-Ehrenmitglied em. Univ.-Prof. Dr. h.c. Dr. Leopold Vietoris. Herr Helmberg hielt die folgende Laudatio.

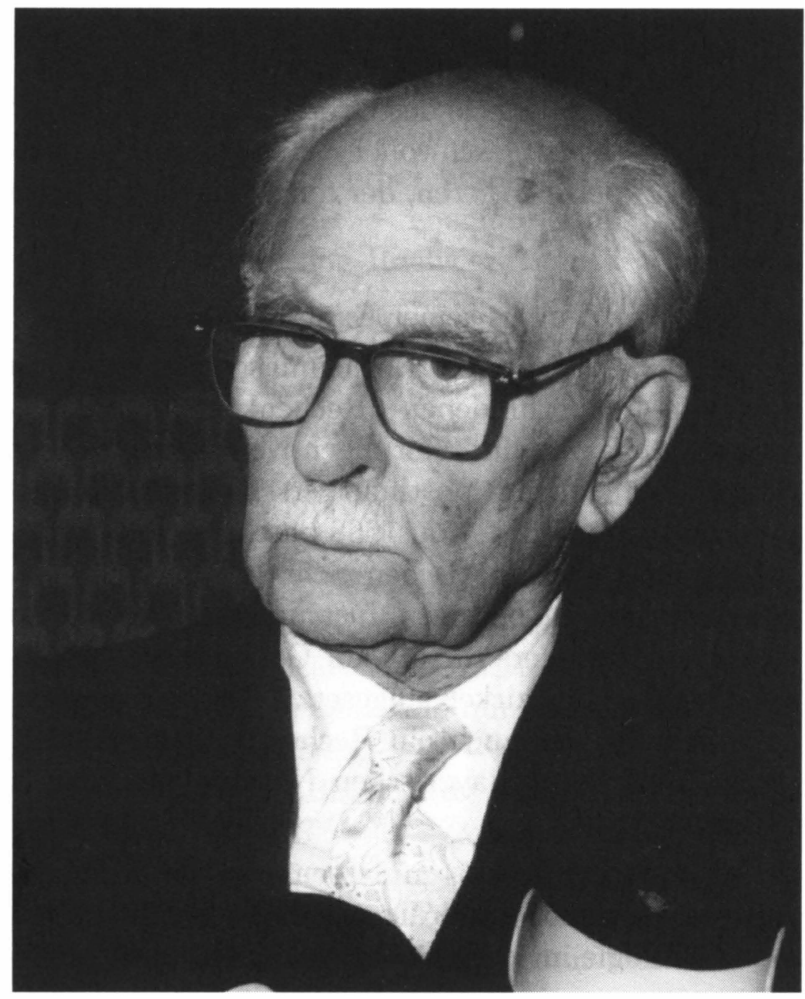

Ein Tisch in einem Konferenzgebäude vor einigen Jahren - ausländische Mathematiker unterhalten sich über ein Fachthema, ein sogenanntes topologisches Trennungsaxiom mit dem Kodenamen T3. Ich höre die Frage eines Kollegen: „Meinen Sie das VietorisAxiom?“ - Erstaunen meinerseits: „Meinen Sie unseren Vietoris in Innsbruck?" - Erstaunen nun bei den Kollegen: „Wie, der Vietoris, auf den dieses Axiom zurückgeht, der ist bei Ihnen in Innsbruck?" — Eine typisch österreichische Situation!

Der Name Vietoris ist heute verbunden mit bedeutsamen mathematischen Begriffen und Erkenntnissen, die als Vietoris-Axiom, Vietoris-Topologie, Vietoris-Homologie, Vietoris-Beglescher Abbildungssatz, Mayer-Vietoris-Sequenz bekannt sind, im Ausland vielleicht mehr als in seiner Heimat.

Im Tiroler Raum ist Vietoris bekannt als Verfasser früher Studien über das Festigkeitsverhalten des alpinen Schis, über die Physik der Blockgletscher, über die Orientierung im Gelände, Studien, in denen Mathematik und Geometrie dienstbar gemacht werden der Lesbarkeit unserer Welt.

Leopold Vietoris wird am 4. Juni 1891 in Radkersburg geboren. Er studiert in Wien Mathematik an der Universität und Darstellende Geometrie an der Technischen Hochschule. Im ersten Weltkrieg wird er im Osten verwundet, aber 1916 erscheint seine erste Arbeit in den Sitzungsberichten der Akademie der Wissenschaften. Am Kriegsende gerät er als HeeresBergführer in Südtirol in italienische Gefangenschaft, aber diese gibt ihm Gelegenheit zum Abschluß seiner Dissertation, die er 1919 zur Erlangung des Doktorates der Philosophie an der Universität Wien einreicht. Nach einem Jahr als Mittelschullehrer erhält er eine Einladung auf die Assistentenstelle am Mathematischen Institut der Technischen Universität Graz. 1923 habilitiert er sich an der Universität Wien, 1925 beginnt er einen dreisemestrigen Forschungsaufenthalt in Amsterdam, 1927 wird er Extraordinarius in Innsbruck, 1928 Ordinarius an der Technischen Hochschule Wien und von 1930 bis zu seiner Emeritierung im Jahre 1960 arbeitet er als Ordinarius für Mathematik an der Universität Innsbruck. Bei der Geburt seiner sechsten Tochter erliegt seine Frau Klara dem Kindbettfieber, ihre Schwester Maria sorgt seit 1936 als seine Gattin für die Familie. Die Österreichische Akademie der Wissenschaften wählt ihn zum wirklichen Mitglied, die Österreichische Mathematische Gesellschaft und die Deutsche MathematikerVereinigung zum Ehrenmitglied, und die Technische Universität Wien verleiht ihm ein Ehrendoktorat.

Die Universität Innsbruck verleiht das heutige Ehrendoktorat an Professor Vietoris in Würdigung seines Lebenswerkes, das mit der Universität Innsbruck eng verbunden ist. Auf die Frage, warum es ein Ehrendoktorat der Technischen und nicht der NaturWissenschaften ist, haben Sie, Professor Vietoris, bereits eine erste Antwort gegeben: Es entspricht universitärer Tradition, daß so eine Ehrung nicht von der eigenen Fakultät kommt. Eine zweite Antwort ist: Sie haben in diesem Lebenswerk abstrakte Forschung und angewandte Wissenschaft verbinden können und angesichts weltweiter Anerkennung Ihres Wirkens damit auch für eine Fakultät, die technische Wissenschaften pflegt, ein Beispiel gesetzt.

Sie haben gezeigt, wie die Mathematik uns immer wieder Einblick in die große Ordnung gewähren kann, die für den gläubigen Menschen Gott in diese Welt gelegt hat, und daß es auf Grund dieser Einblicke möglich ist, einen Beitrag dazu zu leisten, diese Welt in ihrer Vielfalt und ihrer Einmaligkeit zu begreifen eine Aufgabe der Geistes- und Naturwissenschaften und gleichzeitig zu bewahren, lebbar zu machen und lebbar zu erhalten - eine Aufgabe der Technischen Wissenschaften.

Ein Kollege hat kürzlich festgestellt: Vietoris steht über allen Ehrungen. Wir hoffen, diese Ehrung 
macht Ihnen trotzdem Freude. Ich glaube aber, daß wir - die Universität, die Fakultät und ihre Angehörigen - selbst am meisten von dieser Ehrung haben. Wir haben Ehr-Geiz im wörtlichen Sinne: Dafür, daß wir sagen können „Dieser Vietoris ist einer von uns“, möchten wir uns bei Ihnen bedanken mit dem heute zu verleihenden Ehrendoktorat.

Was sind fünf Minuten Laudatio gegenüber einem Lebenswerk eines Jahrhunderts? In der Einsicht, wie hoffnungslos es ist, hier Angemessenes zu sagen, bitte ich Sie: erlauben Sie mir zu schließen mit einem dritten Argument für die Verleihung des Ehrendoktorates auf Antrag einer Fakultät für Bauingenieurwesen und Architektur - mit einem Argument, das hoffentlich dem Lächeln entspricht, mit dem Sie auf vieles zurückblicken, das in Ihrem Leben einmal schwierig erschienen sein mag.

Erst vor kurzem habe ich gehört, daß Sie als Sohn eines Bauingenieurs, der an der Südbahn und der Wiener Hochquellenleitung mitbaute, Ihr Studium an der Technischen Hochschule Wien als Student des Bauingenieurwesens begonnen haben. Sie sind damals nach einem Semester der Faszination der Mathematik und Darstellenden Geometrie verfallen und haben sich in diesen Wissenschaften weltweit einen Namen gemacht. Jetzt hat das Bauingenieurwesen, das Sie damals geglaubt haben, zu verlassen, Sie wieder eingeholt und sagt Ihnen: wir freuen uns, Sie doch auch als einen von uns ansehen zu dürfen.

Beim Autor kann ein VHS-Videoband mit dem Titel „Gespräch mit Leopold Vietoris, 9. Juni 1995“ zum Preis von öS 1500 (zuzüglich Portokosten) angefordert werden.

Adresse des Autors:

Prof. Dr. G. Helmberg

Institut für Mathematik und Geometrie

Universität Innsbruck

Technikerstraße 13

A-6020 Innsbruck

\section{Die Ineffektivität unserer Hohen Schulen}

\section{von Horst Tietz}

Das folgende Referat ist ein Beitrag zur Tagung der „Gesellschaft für Verantwortung in der Wissenschaft e. V.

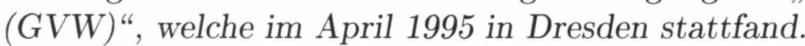

Mein Thema hat durch die Evaluierung der Lehre, über deren Planung seit Monaten berichtet wird, Aktualität erhalten; ich behaupte aber, daß der Riesenaufwand, der hierfür getrieben werden soll, nicht nur, wie ich nachher begründen will, eine Gefahr herauf beschwört, sondern wie bei allen voraufgehenden Reformen, erfolglos bleiben wird, wenn man sich nicht der trivialen Einsicht öffnet, daß das Studium ein Prozeß ist, der zwischen Menschen abläuft. Ohne diese Sensibilität wird der Übelstand nur verschärft werden, daß immer mehr Menschen studieren, immer weniger Erfolg haben - und dann oft erst nach dem Verlust vieler kostbarer Jahre.

Die Atmosphäre ist, besonders im Grundstudium, in hohem Maße lähmend, weil die menschliche Basis für eine geistige Heimat kaum noch vorhanden ist: sie wurde von dem seit 1968 systematisch und genüßlich geschürten Mißtrauen gegen die Hochschulen zerstört, wurde von den Reformen ignoriert und ist im Gestrüpp planerischer Maßnahmen und rechtlicher Bestimmungen zum Surrogat der Gremienarbeit geschrumpft.

Ich möchte nicht mißverstanden werden: es gibt unzählige Ideen, Initiativen und Veranstaltungen, mit denen die der Hochschule angemessene Atmo- sphäre freudiger Geistigkeit demonstriert wird; es muß aber mehr geschehen, wenn solche Angebote zum Konsens führen sollen!

Externe wie Interne müssen der Universität bewußt helfen, für künftige Jahrgänge die alma mater zu sein! Ein schlimmes Indiz für menschliche Kargheit an Universitäten ist die Beobachtung, wie einsam fertige Kandidaten beim Verlassen der Universität sein können ...

Zunächst zwei Worte zur Evaluierung: sie hat für die Forschung einen Sinn - für die Lehre ist sie wertlos, ja sogar gefährlich! Ich denke dabei an meine eigenen akademischen Lehrer: der genialste unter ihnen war didaktisch so hilflos, daß er heute nur kopfschüttelnde Ablehnung auslösen würde; aber sein Ringen um gedankliche Klarheit faszinierte uns so sehr, daß jeder ihm atemlos zu folgen versuchte; in Prüfungen verstand er es, durch die Synthese von wissenschaftlicher Unerbittlichkeit mit begeisternder Menschlichkeit Kandidaten zu Höchstleistungen zu führen. Nach heutigen Vorstellungen würde dieser großartige Mann vermutlich nicht einmal bis zur Habilitation kommen und könnte den Erfolg seiner Lehre durch die hohe Erfolgsquote seiner Prüfungen garnicht demonstrieren. Dagegen führt jede denkbare 